\title{
Insuficiencia aórtica sistólica, un fenómeno singular
}

\author{
Julián Vega ${ }^{1}$, Samuel Córdova ${ }^{1}$, Luigi Gabrielli ${ }^{1,2}$, Rodrigo Saavedra ${ }^{1}$, Paul McNab ${ }^{1}$. \\ 1. División de Enfermedades Cardiovasculares. Pontificia Universidad Católica de Chile. \\ 2. Advanced Center for Chronic Diseases. Escuela de Medicina. Pontificia Universidad Catolica de Chile.
}

Resumen: La insuficiencia aórtica consiste en el reflujo diastólico de sangre desde la aorta hacia el ventrículo izquierdo, sus mecanismos son múltiples y su magnitud queda determinada por: el área del orificio regurgitante, el gradiente diastólico entre la aorta y el ventrículo izquierdo y la duración de la diástole. En contraposición a esto la insuficiencia aórtica sistólica (IAS) es un fenómeno inusual, con escasos reportes y con una fisiopatología particular. Reportamos tres casos clínicos que ilustran los mecanismos de la IAS.

CASO 1: Hombre 54 años, consulta por palpitaciones y disnea, la ecocardiografía transtorácica (ETT) objetiva hipertrofia ventricular izquierda leve con buena función y una valvulopatía aórtica degenerativa con insuficiencia leve, durante el examen se registran extrasístoles ventriculares aislados asociado a IAS.

CASO 2: Hombre de 61 años con diagnóstico de miocardiopatía dilatada no isquémica en estadio D. Su ETT basal evidencia severa dilatación y disfunción ventricular izquierda (FEVI 19\%, DTD 90 $\mathrm{mm}$ ), insuficiencia mitral funcional severa e insuficiencia aórtica leve. Por dependencia a infusión de inótropos se implanta en Marzo del 2016 un dispositivo de asistencia ventricular izquierda HeartWare, en control ambulatorio el dispositivo funcionaba normalmente y el ETT objetiva una significativa disminución de los diámetros del ventrículo izquierdo (DTD $70 \mathrm{~mm}$ ) una válvula aórtica con apertura intermitente asociado a una IAS en rango leve a moderado.

CASO 3: Mujer de 82 años con insuficiencia cardíaca y severa disfunción y dilatación ventricular izquierda, se hospitaliza por infección respiratoria, un ETT muestra insuficiencia mitral funcional severa asociado a IAS moderada. 


\section{Systolic aortic regurgitation: a remarkable event}

Aortic regurgitation is a diastolic event in which blood from the aorta regurgitates back to the left ventricle, with several possible mechanisms. Its magnitude is determined by: the regurgitant orifice area, the diastolic gradient between the aorta and the left ventricle and the duration of diastole. In contrast, systolic aortic regurgitation (SAR) is an unusual phenomenon with few cases reported in the literature and with a particular pathophysiology. We report three cases of SAR that illustrate its mechanisms.

CASE 1: 54 years-old hypertensive male, refers dyspnea associated with irregular heartbeatsTransthoracic echocardiography (TTE) revealed a mild left ventricular hypertrophy with normal function along with a degenerative aortic valve that presented mild regurgitation, isolated premature ventricular contractions were frequently observed associated with mild SAR.

CASE 2. 61 years-old diabetic male, with a long history of stage D dilated non ischemic cardiomyopathy. His last TTE was remarkable for a severe left ventricular dilatation and dysfunction (LVEF $19 \%$, EDD $90 \mathrm{~mm}$ ), severe functional mitral regurgitation, mild aortic regurgitation and pulmonary hypertension (SPAP $60 \mathrm{mmHg}$ ) along with right ventricular dysfunction. Because of inotrope infusion dependency a left ventricular assist device (LVAD) Heartware was implanted. At six months outpatient control, LVAD presented normal function parameters and TTE showed significant reduction in left ventricle diameters (EDD $70 \mathrm{~mm}$ ) along with mild to moderate SAR.

CASE 3: 82 year-old female, longstanding heart failure with severe LV dilatation and dysfunction (LVEF 15\%), admitted for a respiratory infection, TTE showed moderate SAR along with functional severe mitral regurgitation.

Keywords (MeSH): Aortic Valve Insufficiency, Heart Failure, Heart-Assist Devices. 


\section{Introducción:}

La insuficiencia aórtica consiste en el reflujo diastólico de sangre desde la aorta hacia el ventrículo izquierdo y sus mecanismos son múltiples. En vista de una mejor comprensión y manejo se debe considerar a la válvula aórtica como una unidad funcional compuesta por tres estructuras: 1. El anillo aórtico funcional (unión aorto-ventricular y unión sino-tubular), 2. Los velos aórticos con sus sitios de inserción y 3. Los senos de Valsalva. Recientemente la clasificación de El Khoury ${ }^{1}$ sobre la fisiopatología de la insuficiencia aórtica divide sus causas en grupos similares a la clasificación de Carpentier's de la insuficiencia mitral. Existiendo el grupo I: Movimiento normal de los velos, grupo II: prolapso de velos y grupo III, restricción de velos. Cualquier condición que distorsione las estructuras antes mencionadas puede producir insuficiencia aórtica, y su magnitud queda determinada por el área del orificio regurgitante, el gradiente diastólico entre la aorta y el ventrículo izquierdo y la duración de la diástole. En contraposición a esto, la insuficiencia aórtica sistólica (IAS) es un fenómeno inusual, con escasos reportes en la literatura ${ }^{2}$, $3,4,5$ y con una fisiopatología particular que discutimos al final de este artículo. A continuación reportamos tres casos clínicos que ilustran los distintos mecanismos y escenarios clínicos en que se presenta IAS.

\section{CASO 1}

Hombre de 54 años, hipertenso, con historia de palpitaciones episódicas asociado a disminución de la capacidad funcional de I a II NYHA en el último año. Se solicita una ecocardiografía transtorácica (ETT), que objetiva una hipertrofia ventricular izquierda leve con buena motilidad global y segmentaria (FEVI 60\%), una dilatación auricular izquierda y una valvulopatía aórtica degenerativa con insuficiencia leve, durante el examen se registraron extrasístoles ventriculares (EV) monomorfos aislados de forma frecuente, tras lo cual se observó aparición de IAS (Figura 1 y 2). En la señal del Doppler continuo (Figura 2, panel superior) se registró una escotadura o "notch" (asterisco color verde) posterior al EV que coincide con un intento parcial (latido inefectivo) de cerrar la válvula aórtica por parte del ventrículo izquierdo y que genera una disminución transitoria en la velocidad del jet regurgitante. En el panel inferior de la Figura 2 el modo $M$ color permite una mejor apreciación de la temporalidad de la IAS y permite observar la falta de eyección aórtica post EV (latido inefectivo).
Figura 1. ETT. Vista apical de 3 cámaras con Doppler color.

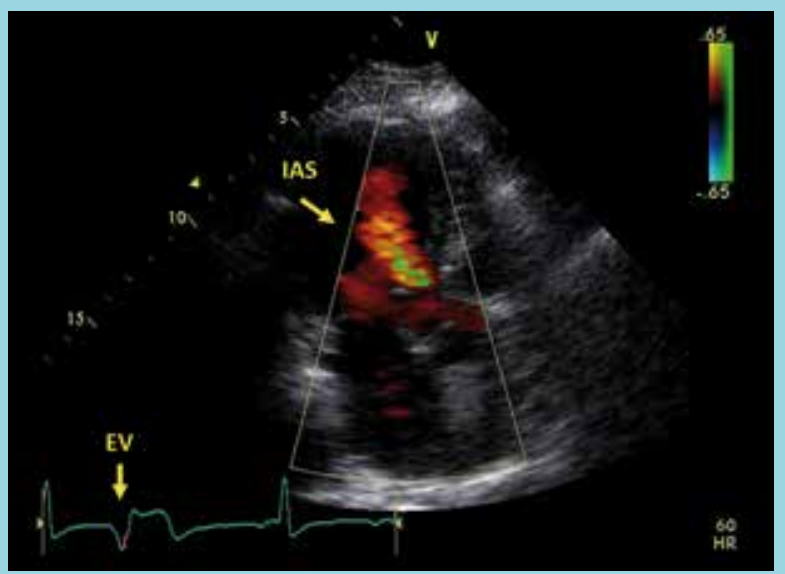

ETT. Vista apical de 3 cámaras con Doppler color. Se observa la presencia de un jet insuficiencia aórtica sistólica (IAS) tras un extrasístole ventricular (EV). Video disponible en http://www.ecocardio.cl/ias.html

\section{Figura 2}

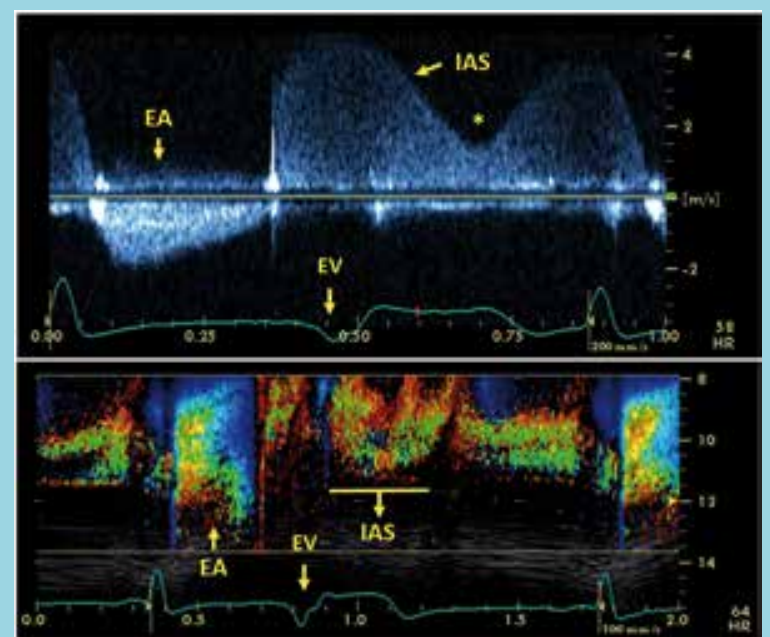

Registro Doppler de IAS. PANEL SUPERIOR: Señal de Doppler continuo sobre la válvula aórtica, evidenciando una insuficiencia continua (sistólica y diastólica) con una escotadura (*) luego del extrasístole ventricular. Se aprecia además la ausencia de eyección aórtica posterior al EV que traduce una contracción ventricular inefectiva. PANEL INFERIOR: Modo M color sobre la válvula aórtica que permite una mejor correlación de los eventos con el ciclo cardíaco.

EA: Eyección aórtica. EV: Extrasístole ventricular. IAS: Insuficiencia aórtica sistólica.

\section{CASO 2}

Hombre de 61 años, HTA, diabético insulino requirente, con diagnóstico de larga data de miocardiopatía dilatada no isquémica en estadio D. El ETT de final del año 2015 mostraba una severa dilatación y disfunción ventricular izquierda (FEVI 19\% por Simpson biplano y diámetro tele diastólico (DTD) de $90 \mathrm{~mm}$ ) asociado a insuficien- 
cia mitral funcional severa, insuficiencia aórtica leve e hipertensión pulmonar de $60 \mathrm{mmHg}$ con disfunción del ventrículo derecho. Por persistir en capacidad funcional IV y dependiente de infusión de inótropos, en Marzo del 2016 se instala como terapia de destinación un dispositivo de asistencia ventricular (DAV) izquierda HeartWare (HeartWare International Inc., Framingham, Massachusetts), siendo dado de alta en buenas condiciones generales. En control 6 meses post implante el DAV evidenciaba parámetros normales de funcionamiento y el ETT evidenciaba una significativa disminución de los diámetros del ventrículo izquierdo (DTD $70 \mathrm{~mm}$ ), un septum interventricular centrado en la línea media, la cánula de succión del DAV se encontraba normo posicionada en el ápex del ventrículo izquierdo (Figura 3 panel A) y la cánula de retorno en la aorta ascendente presentaba flujo laminar. La válvula mitral y tricúspide presentaban ambas insuficiencia leve y la válvula aórtica presentaba apertura intermitente (cada 3 a 4 latidos) asociado a una IAS en rango leve a moderado (Figura 3 ).

\section{CASO 3}

Mujer de 82 años, con diagnóstico de cardiopatía hipertensiva asociado a disfunción ventricular izquierda desde el año 2002. Hospitalizada por insuficiencia cardíaca descompensada asociada a una infección respiratoria. Se solicita un ETT que objetiva un ventrículo izquierdo severamente dilatado (DTD $66 \mathrm{~mm}$, DTD indexado $45 \mathrm{~mm} / \mathrm{m} 2$ ) con hipocinesia difusa y severa disfunción sistólica global (FEVI 15\% por Simpson biplano), dilatación auricular izquierda severa, además de insuficiencia mitral funcional severa y una valvulopatía aórtica

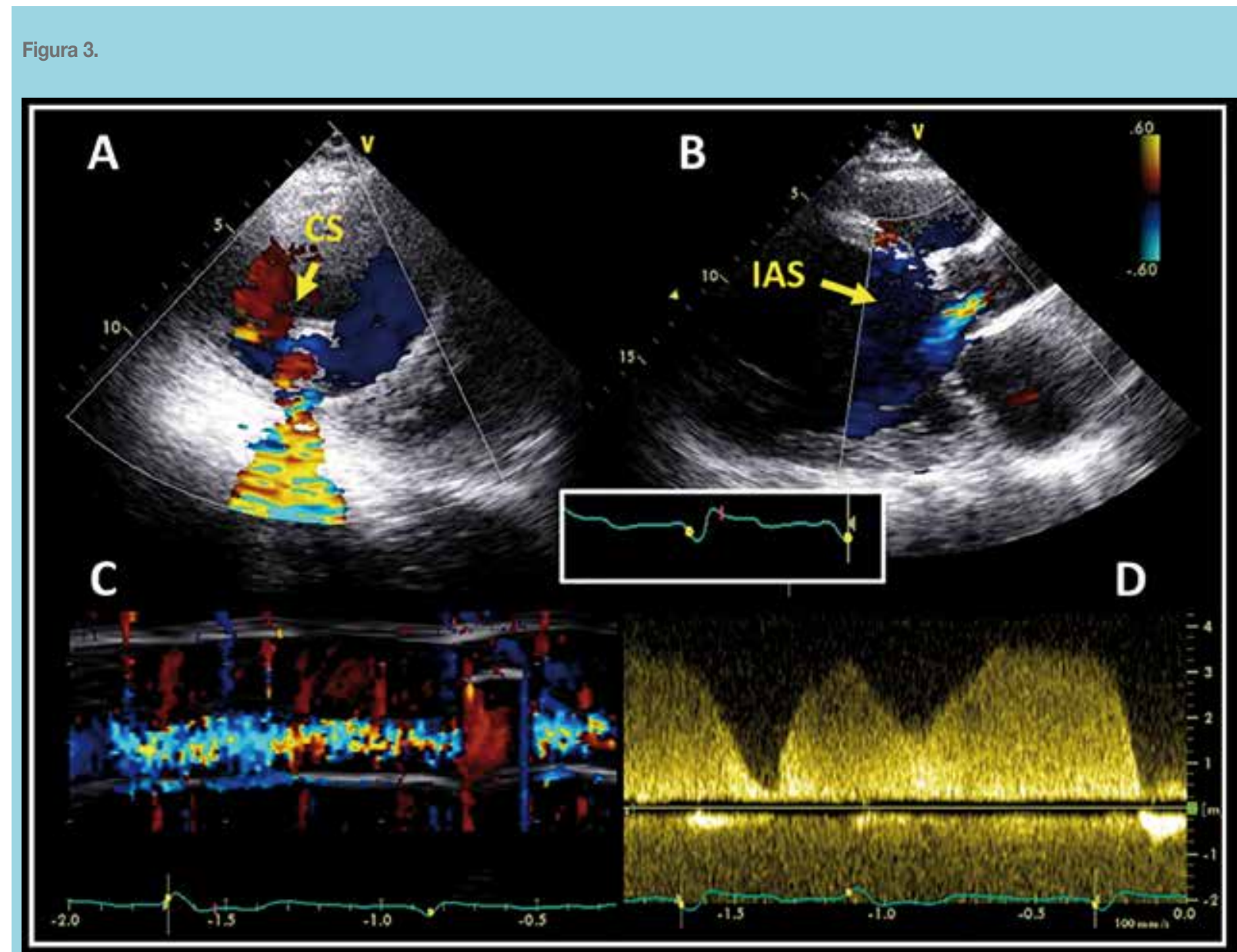

ETT de control post implante de DAV. PANEL A: Eje corto a nivel apical del ventrículo izquierdo, se observa la cánula de succión (CS) del DAV y un artefacto de flujo turbulento al Doppler color generado por el rotor. PANEL B. Eje largo paraesternal izquierdo, que muestra la presencia de IAS en rango leve a moderado. PANEL C: Modo M Color sobre la válvula aórtica, se observa un jet de insuficiencia aórtica continuo durante el ciclo cardíaco. PANEL D: Doppler continuo sobre la válvula aórtica. CS: Cánula de succión. IAS: Insuficiencia aórtica sistólica.

Video disponible en http://www.ecocardio.cllias.html 
Figura 4.

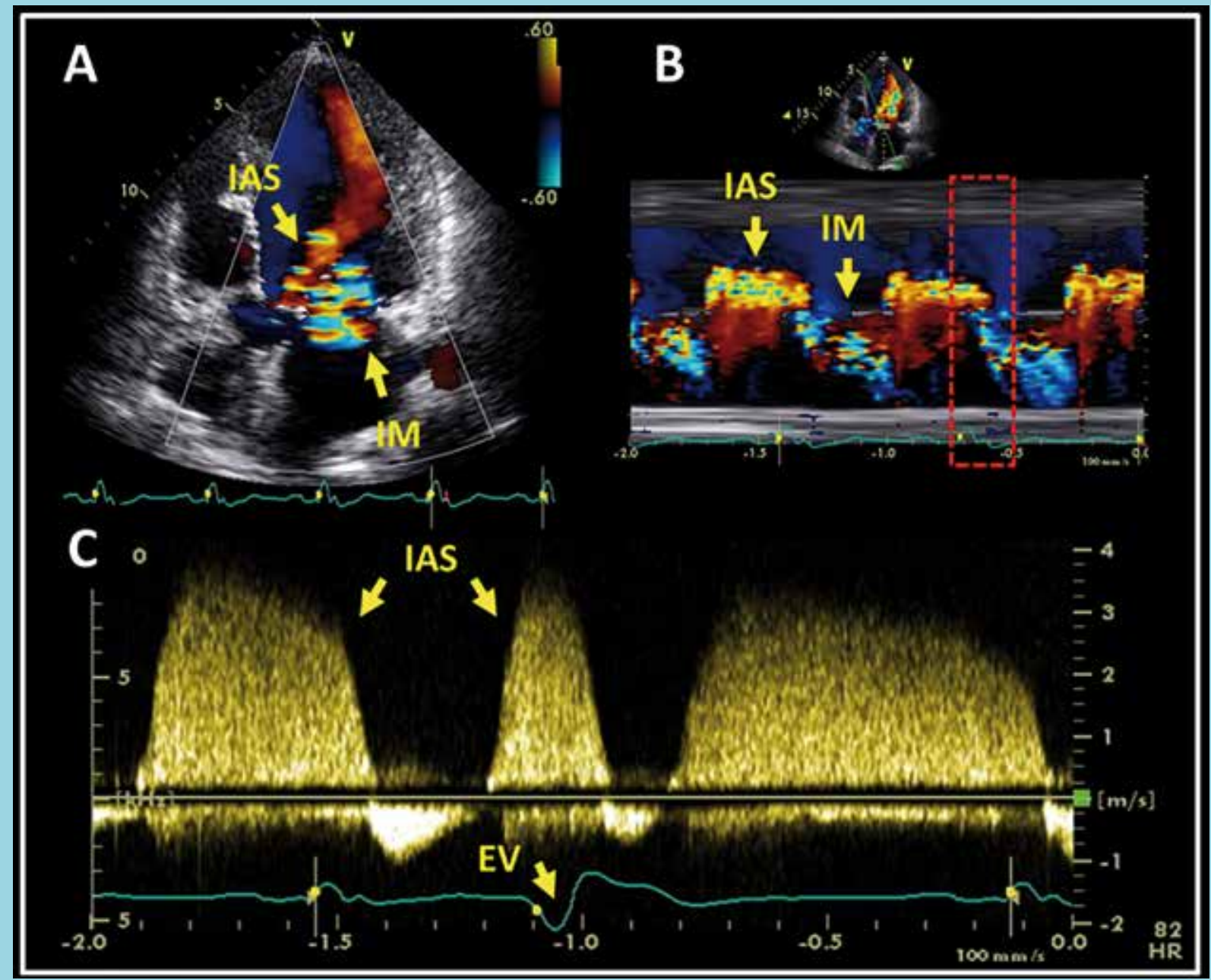

ETT insuficiencia aórtica y mitral sistólica.EL A. ETT vista apical 4 cámaras, se observa en sístole la presencia simultánea de insuficiencia aórtica y mitral. PANEL B: Modo M color se observa en el recuadro rojo la coincidencias de ambas insuficiencias. PANEL C: Doppler continuo sobre la válvula aórtica, se observa un latido sinusal con componente sistólico de insuficiencia aórtica, luego un EV asociado a IAS.

EV: Extrasístole ventricular. IAS: Insuficiencia aórtica sistólica. IM: insuficiencia mitral.

Video disponible en http://www.ecocardio.cl/ias.html

degenerativa con insuficiencia moderada y un componente sistólico permanente. Se registraron además EV aislados frecuentes asociado a IAS. En la Figura 4, panel A, se puede observar en una vista de 4 cámaras con Doppler color la aparición simultánea de insuficiencia mitral y aórtica sistólica.

\section{Discusión:}

La IAS es un fenómeno generado por la incapacidad de un latido ventricular de superar la presión aórtica y para su aparición se requiere, que una vez iniciada la insuficiencia aórtica diastólica habitual se mantenga el gradiente de presión desde aorta hacia ventrículo izquierdo durante la sístole, para esto habitualmente debe existir disfunción ventricular o una baja precarga en el latido previo a la IAS (como el caso de un EV). Para su mejor comprensión en la Figura 5 se presenta un esquema hemodinámico de dicho fenómeno en el contexto de un EV. Esta infrecuente situación tiene una incidencia cercana al $2 \%$ de los pacientes referidos a un ETT y en presencia de insuficiencia cardiaca puede aumentar 


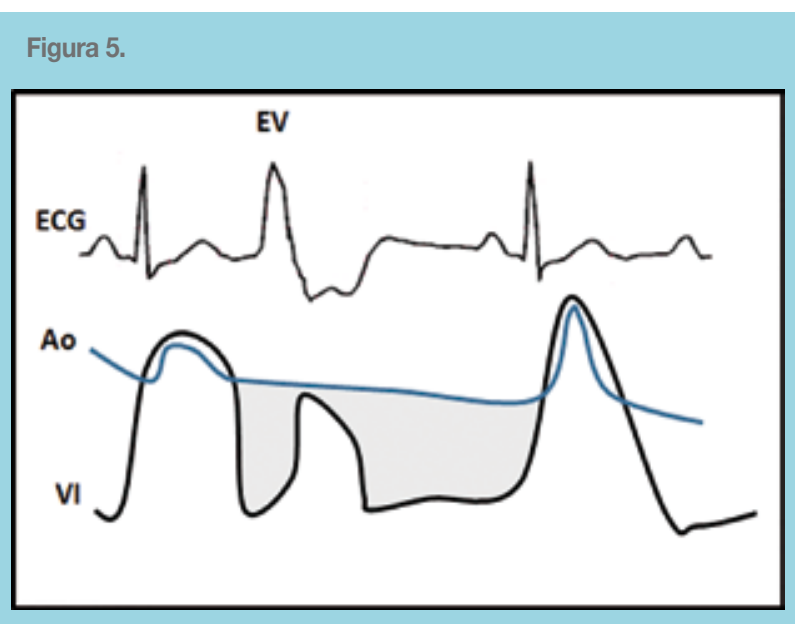

Esquema hemodinámico de IAS asociada a EV . PANEL SUPERIOR: Trazado de ECG con un EV aislado. PANEL INFERIOR: Curvas de presión de la aorta (Ao) y del ventrículo izquierdo (VI). Luego del EV se observa una contracción ventricular que alcanza menor presión y no logra superar la presión aórtica para generar apertura valvular, lo que se traduce en una disminución del gradiente y es la causa de la escotadura registrada en el Doppler continuo del ETT. El área en gris representa el gradiente de presión a favor de la aorta que se mantiene durante el latido post EV y es la que genera la insuficiencia aórtica sistólica. hasta tres veces ${ }^{6}$. La IAS ha sido descrita en casos de disfunción ventricular izquierda, fibrilación auricular, posterior a reparaciones complejas de cardiopatías congénitas y luego de una contracción ventricular inefectiva asociada a extrasístoles ventriculares, situación en la que fueron descritos los primeros reportes de dicho fenómeno ${ }^{3}$. $\mathrm{Al}$ anterior mecanismo se adiciona en el último tiempo el relacionado a los DAV de flujo continuo, que causan IAS mediante varios mecanismos, siendo uno de ellos la succión permanente desde el ápex del ventrículo izquierdo. Se debe considerar que la presencia de insuficiencia aórtica significativa es perjudicial para el rendimiento del $\mathrm{DAV}^{7}$, al generarse un circuito cerrado tipo "loop" entre la aorta ascendente y el ventrículo izquierdo disminuyendo la perfusión sistémica, requiriendo por tanto, corregir dicha insuficiencia según sea su magnitud en el momento del implante del dispositivo $^{8}$. Finalmente la IAS es una situación excepcional que al igual que la insuficiencia mitral diastólica, nos permiten incrementar nuestra compresión de la fisiopatología cardíaca.

\section{Referencias}

1. DE KERCHOVE L, EL KHOURY G. Anatomy and pathophysiology of the ventriculo-aortic junction: implication in aortic valve repair surgery. Ann Cardiothorac Surg. 2013;2: $57-64$.

2. SHETTY V, LODHA A, MOHAMED E, SADIQ A, SHANI J. Systolic aortic regurgitation: A rare phenomenon. Int J Cardiol. 2010;144: e58-60.

3. KRONZON I, KONECKY N, TUNICK PA. Systolic Aortic Regurgitation : A Hemodynamic Challenge for the Clinician. J Am Soc Echocardiogr. 1995;8: 941-943.

4. UNGER P, VANDENBOSSCHE J-L. Doppler demonstration of systolic aortic regurgitation. Am Heart J. 1992;123: 805807.

5. SAURA D, GONZÁLEZ J, DE LA MORENA G, VAL-
DÉS-CHÁVARRI M. Systolic aortic regurgitation. Eur J Echocardiogr. 2008;9: 284-5.

6. SAURA D, PEÑAFIEL P, MARTÍNEZ J, DE LA MORENA G, GARCÍA-ALBEROLA A, SORIA F, et al. The frequency of systolic aortic regurgitation and its relationship to heart failure in a consecutive series of patients. Rev española Cardiol. 2008;61: 771-774.

7. COWGER J, PAGANI FD, HAFT JW, ROMANO MA, AARONSON KD, KOLIAS TJ. The development of aortic insufficiency in left ventricular assist device-supported patients. Circ Hear Fail. 2010;3: 668-674.

8. HOLTZ J, TEUTEBERG J. Management of aortic insufficiency in the continuous flow left ventricular assist device population. Curr Heart Fail Rep. 2014;11: 103-10. 